\title{
Improving design of a PVDF grain loss sensor for combine harvester
}

\author{
Liming Zhou, Yanwei Yuan ${ }^{(凶)}$, Junning Zhang, Kang Niu \\ Chinese Academy of Agricultural Mechanization Sciences, Beijing 100083, China \\ \{haibo1129, yyw215, zjn990210\}@163.com, 715675915@qq.com
}

\begin{abstract}
Online monitoring of grain loss is important to improve the efficiency of combine harvester. The traditional grain loss sensor needs to be frequently calibrated for different materials. In order to solve this problem, the grain loss sensor was developed based on PVDF (Polyvinyliden Fluoride) films. The band-pass filter based on LTC1068-200 was designed by frequency spectrum analysis of the wheat and rice grain impact response. And the dynamic voltage comparator with adaptive regulation of the threshold voltage according to the intensity of sensor signal was introduced here. Wheat and rice grain samples with different moisture content were prepared for the experiments and the results indicated the sensor had high measurement accuracy to the different samples.
\end{abstract}

Keywords: combine harvester, grain loss sensor, band pass filter, adaptive threshold voltage

\section{Introduction}

Grain loss is the key index for measuring the operating performance of the combine harvester. Traditional measurement method is to obtain the grain from material manually [1-2]. With the development of sensors, electronics and information process technologies, the demand for precise and intelligent detection system for grain loss are rising. Online grain loss monitoring is important to improve harvest efficiency and quality operations.

At present, most of the grain loss monitoring system based on PZT, are to detect the number of passing grain kernels per unit time at the discharge end of the walker [3-7]. Liang proposed an impact type piezoelectric sensor for grain sieve loss monitoring, the signal conditioning circuits were developed to discriminate for full grains [8-9]. But, it is very difficult to fit the different type of grains (such as rice and wheat) adaptively because of the fixed threshold voltage of the signal processing circuit. Since the kernel of grain is much harder than the straw, acoustic sensor had been used to detect the grain loss of harvester. The sound from the sensor was picked up by a microphone firstly, then the signal was processed by a computing device and displayed on a electric meter terminal. But the accuracy of this acoustic grain loss sensor is severely constrained by working conditions, and the noise is a fatal factor for the acoustic sensor [10]. Microwave sensor was also used to detect the grain loss [11]. An array sensor made from PVDF film is designed to determine the grain loss [12].In this paper, how to design the effective signal conditional circuit of the PVDF grain loss sensor was introduced to improve the resolution between the grain and stems. 
The grain impact signals were analyzed for designing a high order band pass filter to discriminate grain online. In order to make the sensor fit rice or wheat grain automatically, the voltage compactor with adaptive threshold was used in this paper. Within the system, the grain loss for rice and wheat was determined and the precision of the method was tested.

\section{Materials and Methods}

\subsection{Fabrication of the PVDF sensor}

The grain loss measuring system consists of the PVDF sensor array, signal conditioning circuit, communication interface and display meter. The array sensor was made from PVDF film, a new piezoelectric material, which was used broadly in the measuring sensor field recently. Compared with the piezoelectric ceramics, it had higher piezoelectric voltage coefficient and better responsive speed. The sensor array was made up of five cells, the size of every cell was $20 * 100 * 0.05 \mathrm{~mm}$, The PET film was affixed to the upper and lower surfaces to protect the sensor unit. In order to add the fuse, the foam rubber double-sided sealing tape was used to attach the sensor to the Aluminum support substrate. At the same time, the sealing tape can improve the deformation of the sensor and enhance the output signal. The frame is shown as Fig.1.

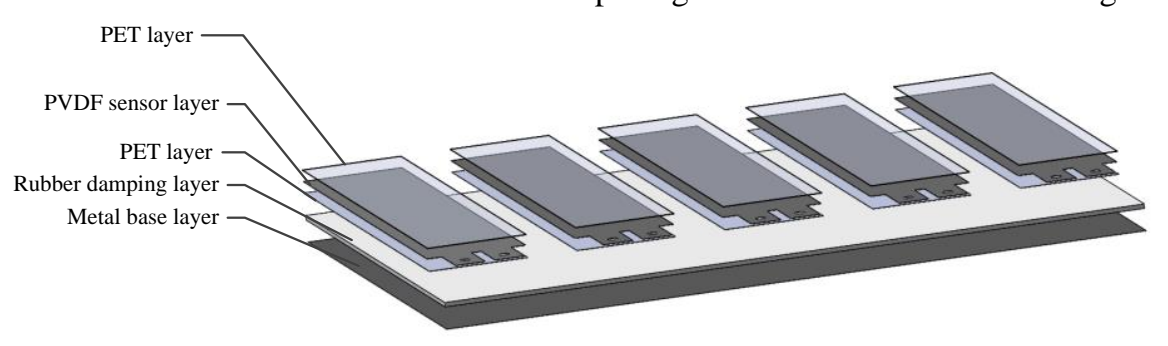

Fig.1. PVDF sensor structure

\subsection{Conditioning circuit}

The conditioning circuit was mainly used to deal with the weak charge signal generated by the PVDF sensor. The circuit was contained of charge conversion amplifier, voltage follower, high order band-pass filter, diode detection, adaptive voltage comparison module and counter. The structure is shown in Fig2.

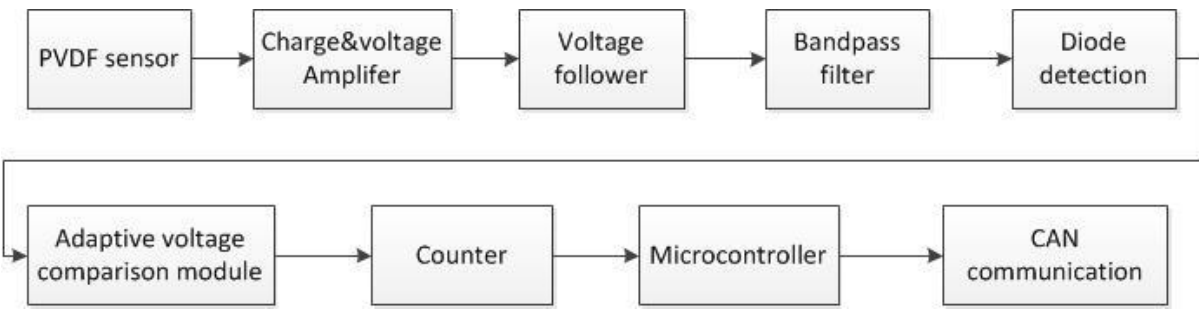

Fig.2. Structure diagram of the signal conditioning circuit 
Firstly, the charge conversion amplifier transforms the initial weak signal generated by the PVDF sensor into the voltage signal, and the amplitude of the voltage signal is between $-4.5 \mathrm{~V}$ and $4.5 \mathrm{~V}$. The signal is subjected to impedance conversion by a voltage follower to reduce the output impedance of the front stage circuit. Meanwhile, the pre-stage circuit is isolated from the post-stage circuit by means of the voltage follower. After band pass filtering, the material other than grain signals are weakened. The output signal through a diode detector which is a diode connected to a resistor and capacitor in parallel, and the envelope of the original signal was obtained. Schmitt trigger is used to transfer the envelope signal to rectangular digital pulse at the same frequency as the input. The trigger has a small amount of hysteresis that will help with noise rejection. The threshold of the trigger can adapt to input signal amplitude.

In this paper, the filter circuit and the voltage comparison circuit have been improved on the basis of the previous work. A band pass filter based on integrated chip was designed to improve the interference immunity of mechanical vibration and electricity. Meanwhile, the dynamic adjustment circuit of Schmitt Trigger threshold based on kernel signal was designed so that the threshold value of grain pulse could be adjusted automatically according to the signal envelop voltage.

\subsection{Bandpass filter circuit}

The amplified sensor signal often contains a variety of frequency band information. And the active filter circuit based on operational amplifier is usually used to discriminate the different frequency signal. In the previous experiment, it was found that the low order band pass filter was not well eliminated by the attenuation rate. If a high-order filter is designed based on an operational amplifier, the increased complexity of the circuit may adversely affect system reliability and stability. In addition, because of the influence of environmental factor, the filter performance may drift. Therefore, LTC-1068-200 can be applied to form eight-order Chebyshev band pass filter. The filter can emphasize seed signals in certain frequency ranges and reject signals in other frequency ranges. LTC1068-200 contains four high accuracy $2^{\text {nd }}$ order switched capacitor filter section.

In order to determine the reasonable parameters of the filter and ensure that the sensor has good application to wheat and rice grain, it is necessary to analyze the signals response of grain seed. Firstly, the sensor output signal was taken through a 200 amplifier, then to a Textronix TDS3000C oscilloscope, the scope's digitized signal was sent to a Matlab R2008b program for data collection and processing. The Fast Fourier Transform were calculated using Matlab signal processing toolbox. The time and frequency response are shown in Fig.3 and Fig.4. 


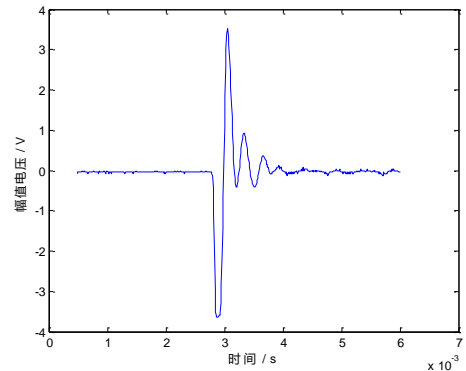

output signal

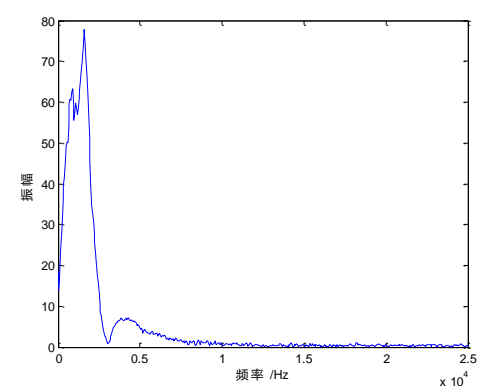

(b) frequency spectrum

Fig.3. Output voltage signal and its FFT transfer of wheat grain

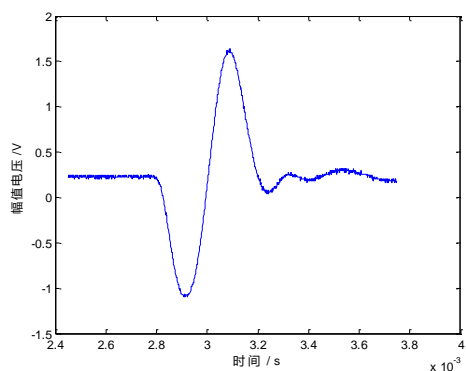

(a) output signal

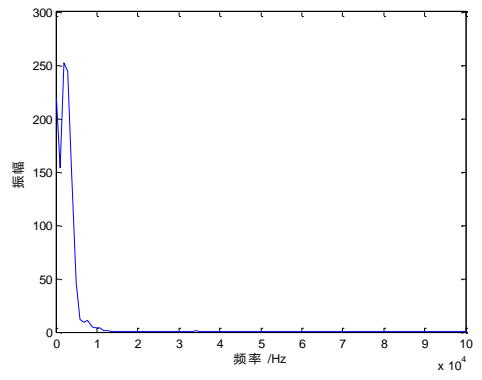

(b) frequency spectrum

Fig.4. Output voltage signal and its FFT transfer of rice grain

The curves in Fig.3(a) and Fig.4(a) are the response signal generated by the wheat and rice grain impact sensor. As can be seen from the curves, the voltage amplitude of wheat signal is about $3.5 \mathrm{~V}$, and that of rice signal is about $1.7 \mathrm{~V}$. In Fig.3(b) and Fig.4(b), we find that the center frequency of rice signal is $1.67 \mathrm{kHz}$ and that of rice signal is $1.95 \mathrm{kHz}$. Obviously, the amplitude and characteristic of two kind signals are different.

According to the above analysis, it is determined that the center frequency of the designed eight order Chebyshev band pass filter is $1.81 \mathrm{kHz}$. The filter bandwidth is $800 \mathrm{~Hz}$. Thus the range of frequencies passed by the filter is $1.41-2.21 \mathrm{kHz}$. The LTC1068-200 requires an external clock signal, which is usually obtained by crystal. This circuit is relatively complex, and also difficult to fine tune the frequency. The microcontroller STC11F02 was used as a signal generator to generate the desired pulse signal and was output by the CLKOUT pin, then the standard clock signal was sent to LTC1068. The eight order Chebyshev band pass filter was designed using Linear Technology's FilterCAD for Windows. Design of the filter is very easy with the help of this program. Fig.5 shows the bandpass filter based on the LTC1068-200 with $\pm 5 \mathrm{~V}$ power supplies. In order to keep the impedance matching of the circuit, a voltage follower based on LT1355 was used here. The gain response curves of the band pass filter is shown in Fig.6. 


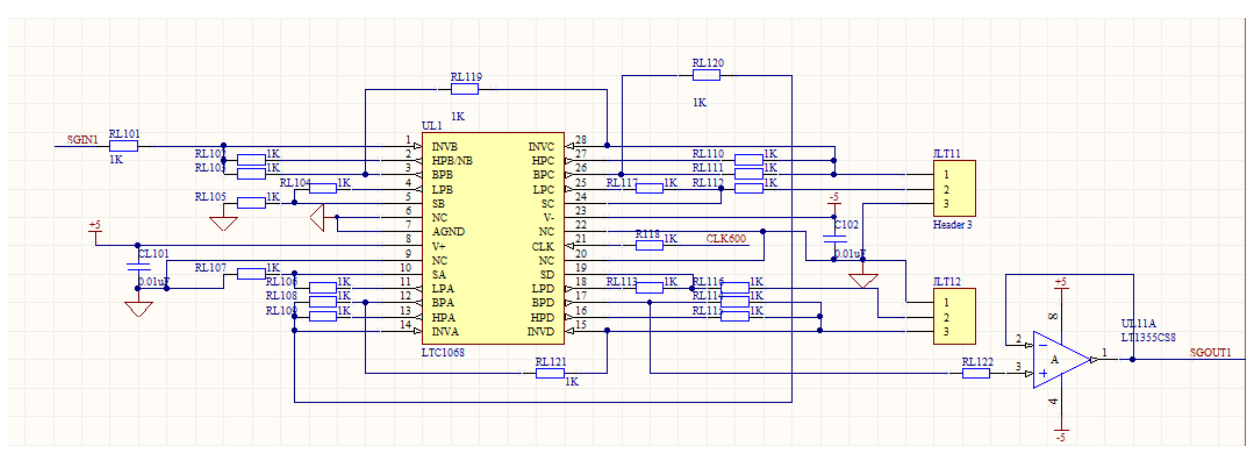

Fig.5. Schematic of the Chebyshev filter

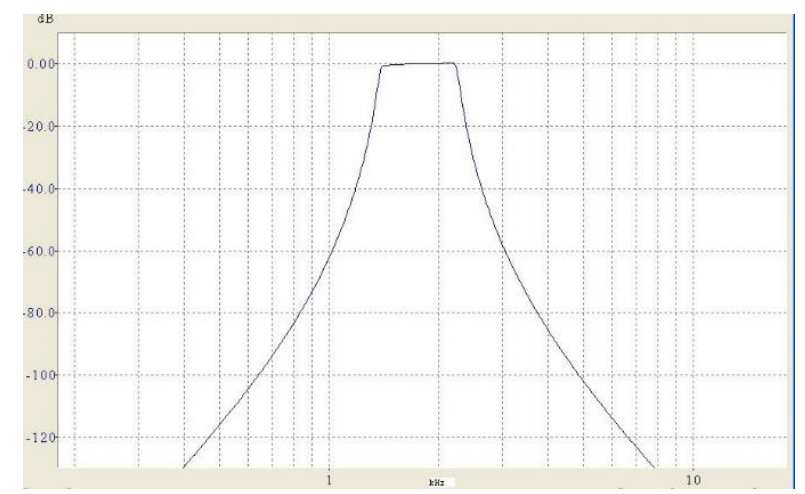

Fig.6. Frequency response of the designed filter

It was observed that bandwidth of the designed filter in the $-3 \mathrm{db}$ was not greater than $800 \mathrm{~Hz}$ in Fig.6. It means that the filter can work very well. Meanwhile, the output signal of the filter was connected to the diode detector circuit in order to obtain the envelope of the grain signal.

\subsection{Self-adaptive voltage compactor}

After the envelope detecting, the pulse shaping circuit for active counting of grain signals was introduced here. The Schmitt trigger circuit was designed to obtain a standard square wave signal. Before the Schmitt trigger was used, the hysteresis and the threshold should be determined firstly. Due to the differences in the grain quality of different breeds and moisture, the amplitude of the signal produced by the impact sensor was also different. Meanwhile the amplitude of signal was also affected by the angle and height of the grain drop. In this case the amplitude are variable, setting the thresholds to produce reliable triggering can be tricky [13]. Therefore, we proposed a signal shaping method based on the voltage comparator threshold, and designed a dynamic threshold adjustment circuit in this paper. With this circuit, the threshold voltage in the signal shaping can be adaptively adjusted to improve the signal shaping effect. The circuit schematic is shown in Fig.7. 


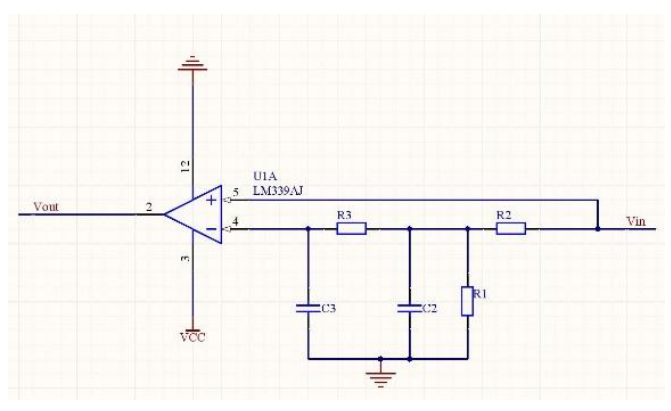

Fig.7. Adaptive threshold voltage comparator

The enveloped module output was divided into two parts, one part was transferred into the positive input of Schmitt trigger, the other was connected to a second order low pass filter which was consisted of Resister R2, R3 and Capacitor C2,C3. After the input signal $V_{\text {in }}$ was filtered, the output DC signal associated with the input signal can be obtained. We can see that the resistor R1 and R2 form a voltage divider, thus the output voltage which is taken as the threshold of the trigger is a fraction of the input signal $V_{\text {in. }}$ In other words, the threshold voltage of the compactor is matched to the input signal $\mathrm{V}_{\text {in }}$ adaptively. So, the accuracy of the comparator output was improved.

\subsection{Counting and communication}

The electronic circuits design for the counting and communication is illustrated in Fig7. The circuit board consisted of the CAN bus controller, Counter and Microcontroller unit (MCU). The standard pulse signal was connected to counter 82C54. The $82 \mathrm{C} 54$ is a high CMOS programmable interval timer, which has three independently programmable and functional 16-bit counters, each capable of handling clock input frequencies of up to $8 \mathrm{MHz}$. The interface using a bidirectional 8-bit ports on the microprocessor gives full control of the timer. Grain loss quantity is obtained by counting the number of pulse with use of counter of $82 \mathrm{C} 54$ for an operator set time period. The single chip STC12C5A60S2 was chosen as the monitor core of the system. The microcontroller provides a full duplex serial, SPI(Serial Peripheral Interface bus) and input/output module.

The MCP2515 was used in the grain loss monitoring sensor for data communication. It handles all of the transmitting and receiving of message packets that contain grain loss information via the CAN bus. The MCP2515 was configured and operated through a Serial Peripheral Interface (SPI) with a microcontroller. At the same time, the TJA1050 transceiver modules was also used as the interface between the MCP2515 CAN bus controller and the physical bus. The device provided differential transmit capability to the bus and differential receive capability to the CAN controller. In order to improve the performance of data communication, the optocoupler 6 N137 was used to isolate the signals. 


\subsection{Software design of the system}

Keil C51 was used to develop the MCU program. Fig.8 shows the program flow for the monitoring system. The software of the sensor was composed of the initialization, counting and data communication. Initialization included initialization of the input/output ports, SPI, counter and CAN module control register setup and reset all related memory locations that were going to be used in the operation. The counter of microcontroller was used to measure the grain seed pulse rate per second. Data communication was used to transfer measured grain loss quality from the sensor to the upper machine.

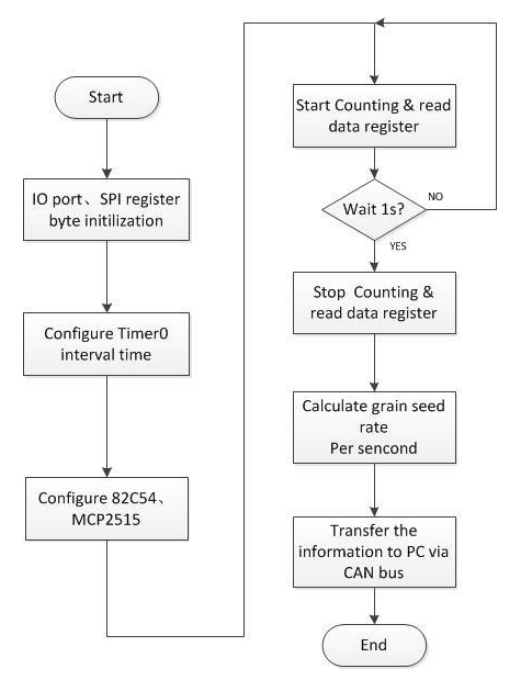

Fig.8. Flowchart of the microcontroller

\section{Results and discussion}

\subsection{Sensor reliability test}

In order to determine the accuracy of grain loss sensor, two rice grain samples with moisture content of $18.3 \%, 23.2 \%$ and two wheat samples with moisture content of $19.1 \%, 25.3 \%$ were used in laboratory test. A total of 12 tests were conducted that included two type of grain and for two moisture contents. The fix angle and height of sensor in the experiments were same. The experiment instruments are shown in Fig.9. 


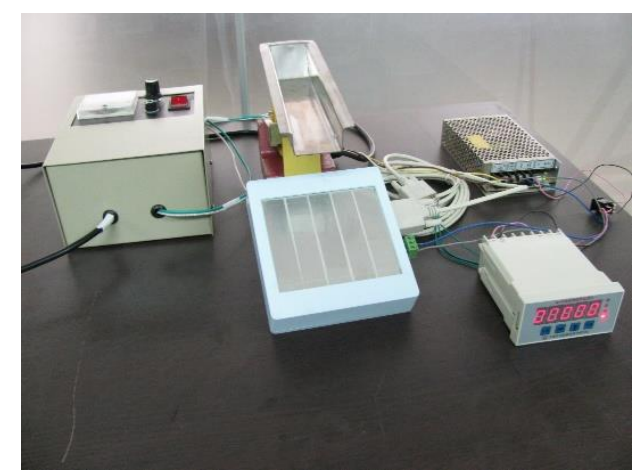

Fig.9. Experiment instruments including PVDF sensor and vibration supplier

At the start of the experiment, the grains were put into electromagnetic vibration supplier, and the exciting current was regulated to keep the drop flow at 100grain/s. Experiments were repeated three times for each sample.

Table1. Test results of grain loss obtained by sensor and manual

\begin{tabular}{|c|c|c|c|c|c|c|c|c|}
\hline \multirow[t]{2}{*}{$\begin{array}{l}\text { Test } \\
\text { No. }\end{array}$} & \multicolumn{2}{|c|}{$\begin{array}{l}\text { Rice grain } \\
(\mathrm{MC}=18.3 \%)\end{array}$} & \multicolumn{2}{|c|}{$\begin{array}{l}\text { Rice grain } \\
(\mathrm{MC}=23.2 \%)\end{array}$} & \multicolumn{2}{|c|}{$\begin{array}{l}\text { Wheat grain } \\
(\mathrm{MC}=19.1 \%)\end{array}$} & \multicolumn{2}{|c|}{$\begin{array}{l}\text { Wheat grain } \\
(\mathrm{MC}=25.3 \%)\end{array}$} \\
\hline & $\begin{array}{l}\text { Measured } \\
\text { value }\end{array}$ & $\begin{array}{l}\text { Relative } \\
\text { error } \%\end{array}$ & $\begin{array}{l}\text { Measured } \\
\text { value }\end{array}$ & $\begin{array}{l}\text { Relative } \\
\text { error \% }\end{array}$ & $\begin{array}{l}\text { Measured } \\
\text { value }\end{array}$ & $\begin{array}{l}\text { Relative } \\
\text { error } \%\end{array}$ & $\begin{array}{l}\text { Measured } \\
\text { value }\end{array}$ & $\begin{array}{l}\text { Relative } \\
\text { error \% }\end{array}$ \\
\hline 1 & 989 & 1.1 & 961 & 3.9 & 983 & 1.7 & 979 & 2.1 \\
\hline 2 & 952 & 4.8 & 959 & 4.1 & 975 & 2.5 & 1022 & 2.2 \\
\hline 3 & 1039 & 3.9 & 982 & 1.8 & 990 & 1.0 & 1017 & 1.7 \\
\hline
\end{tabular}

Table1 shows the relative error analysis of the results obtained by the developed sensor and manual under the same condition. From Table1, it can be seen that the sensor can count the number of grain with the maximum measurement error of $4.8 \%$ for rice grain and that of $2.5 \%$ for wheat grain. The experiment results also show that the sensor has good measurement effect on wheat and rice grains under the same experimental conditions.

\subsection{Discriminability of the different material}

To verify the performance of the conditioning circuit, experiments were conducted with different materials. Three different materials (rice grain, hollow kernel and straw) were taken as experiment samples to test the effect of the sensor to distinguish seed from the materials. Thousand seeds mass of the grain mentioned here was $32.1 \mathrm{~g}$. Firstly, the grain, hollow kernel and straw mentioned before were put into electromagnetic vibration supplier separately. Then the exciting current of supplier was regulated to keep the drop flow at a suitable value. The measured value and actual value were compared here.

Table2. Results of detecting grain and other materials

\begin{tabular}{llll}
\hline & Rice grain & Hollow kernel & Straw \\
\hline Measured amount & 971 grains & $0.85 \mathrm{~g}$ & $22 \mathrm{~g}$ \\
Actual amount & 1000 grains & $0.028 \mathrm{~g}$ & $0.336 \mathrm{~g}$ \\
Misrecognition error & $2.9 \%$ & $3.29 \%$ & $1.53 \%$ \\
\hline
\end{tabular}


Table2 indicated that the sensor was sensitive to the grain and the misrecognition ratio was about $2.9 \%$. It is insensitive to the hollow kernel and straw, the misrecognition ratio is in $4 \%$. Thus the sensor can distinguish the grain from other material very well.

\section{Conclusions}

In this study, the grain loss sensor based on PVDF film was present. The characteristic spectrum of rice and wheat grain was obtained based on the analysis of the frequency response of sensor. A signal conditioning circuit which included a Chebyshev bandpass filter with cutoff frequencies of $1.41-2.21 \mathrm{kHz}$ and Schmitt voltage compactor with adaptive threshold was designed here to improve the detection accuracy and versatility. The test results show that the designed sensor still has a high detection accuracy after changing the material type without calibration. The maximum relative error of wheat and rice is $2.5 \%$ and $4.8 \%$, and the grain can also be distinguished from the hollow kernel and straw effectively.

\section{Acknowledgment}

This research was funded by the National Key Research and Development Program of China (2016YFD0700305) and Science \& Technology Nova Program of Beijing (Z1511000003150116) and Technology developing and achievement transformation project of XPCC(2015AC009).

\section{References}

1. Jie Zhan, Liu Hongjun, Hou Fengyun.: Research advances and prospects of combine on precision agriculture in China. Transactions of the Chines Society of Agricultural Engineering.21(2), 179-182(2005).

2. Zhao Zhan, Yaoming Li, Jin Chen, et al.: Grain separation loss monitoring system in combine harvester. Computers and Electronics in Agriculture.76,183-188(2011).

3. Li Junfeng, JieZhan.: Analyses of combine harvester grain loss measurement. Agricultural Mechanization Research.12, 248-250(2007).

4. Osselaere,Guy H. J.: Offset grain loss sensor for combine harvesters. US Patent 4540003,(1985).

5. Li Yaoming, Chen Yi, Zhao Zhan.: Monitoring method and device for cleaning loss of combine harvester.Transactions of the Chinese Society for Agricultural Machinery.44(S2), 7-11(2013).

6. Ni Jun, Mao Hanping, Li Pingping.:Intelligent grain cleaning losses monitor based on array piezocrystals. Transactions of the Chinese Society for Agricultural Machinery.41(8),175-177(2010).

7. Mao Hanping, Ni Jun:Finite element analysis measurement for array piezocrystals grain losses sensor. Transactions of the Chinese Society for Agricultural Machinery.39(12),123126(2008).

8. Zhenwei Liang, Yaoming Li, Lizhang Xu,et al.:sensor for monitoring rice grain sieve losses in combine harvesters. Biosystems Engineering. 147,51-66(2016)

9. Zhenwei Liang, Yaoming Li, Lizhang Xu,et al.: Optimum design of an array structure for the grain loss sensor to upgrade its resolution for harvesting rice in a combine harvester. Biosystems Engineering. 157,24-34(2017). 
10. Liu C. , Leonard J.:Monitoring actual grain loss from an axial flow combine in real time. Computers and Electronics in Agriculture. 9(3), 231-242 (1993).

11. Kotyk, Wayne M. Kirk, Thomas G. Unthreshed head grain loss monitor.US Patent, 4825146 (1989).

12.Zhou Liming, Zhang Xiaochao, Liu Yangchun, et al.:Design of PVDF sensor for grain loss measuring. Transactions of the Chinese Society for Agricultural Machinery. 41(6), 167171(2010).

13. Deniz Yilmaz, Huseyin Caner Sagiroglu.: Development of measurement system for grain loss of some chickpea varieties. Measurement, 66, 73-79(2015). 